\title{
A Semantic Framework for Assistive Technologies Description to Strengthen UI Adaptation
}

\author{
Nikolaos Kaklanis, Konstantinos Votis, \\ Konstantinos Giannoutakis, and Dimitrios Tzovaras \\ Information Technologies Institute, Centre for Research and Technology Hellas, \\ Thessaloniki, Greece \\ \{nkak, kvotis, kgiannou, Dimitrios.Tzovaras\}@iti.gr
}

\begin{abstract}
The present paper presents the Semantic Framework for Content and Solutions, an ontological framework that enables the classification of various assistive technologies (both software and hardware) according to well-known accessibility standards, such as the ISO 9999 standard, and also the description of all the supported adjustments/settings and their alignment with similar settings of other technologies. This semantic framework is a key component of the whole Cloud4all/GPII infrastructure and strengthens the UI adaptation process as it enhances the inference capabilities of the Rule-based Matchmaker, another component of the Cloud4all/GPII infrastructure that tries to match user needs with the corresponding configurations of different assistive technologies using rules.
\end{abstract}

Keywords: Semantic alignment, ontology, assistive technologies, application classification.

\section{$1 \quad$ Introduction}

Ontologies can play a very crucial role in strengthening the accessibility of user interfaces (UIs). The appearance of the parts of a UI as well as their functionalities may be directed by ontologies. If both UI elements and user needs and preferences are defined within ontologies, a reasoner can determine the appearance that fits user needs best [12]. The mapping between user impairments and user interface characteristics can be also expressed semantically [8].

A typical problem that often appears in ontology-based systems includes the lack of interoperability between other similar systems or even sub-components of the same framework, due to different representations of identical terms. Ontology alignment in the sense of identifying relations between individual elements of multiple ontologies is a necessary precondition to establish interoperability between agents or services using different individual ontologies [3]. Moreover, the semantic alignment enables the searching or browsing of the knowledge represented in numerous ontologies in a transparent way for the end-users. 
Even if many approaches have been proposed for improving the visualization [13] and interaction capabilities [4, 6] of user interfaces by using ontologies, there is a need for an ontological framework that will enable the classification of the most common assistive technologies (both software and hardware) according to wellknown accessibility standards and also the description of all the supported adjustments/settings and their alignment with similar settings of other technologies. The present paper presents the Semantic Framework for Content and Solutions (SEMA) developed within the premises of the Cloud4all FP7 EC project that fulfills the aforementioned requirements. The SEMA is a key component of the whole Cloud4all/GPII infrastructure and strengthens the UI adaptation process as it enhances the inference capabilities of the rule-based matchmaker, another component of the Cloud4all/GPII infrastructure that tries to match user needs with the corresponding configurations of different assistive technologies using rules.

\section{$2 \quad$ Related Work}

There are several efforts towards the direction of defining ontological concepts and architectures for the semantic representation of ICT solutions, devices and platforms within the area of e-Inclusion and personalised interfaces. These efforts try to cover adequately the personal requirements of the end users, under an ICT artifact specific perspective. For example, besides user modeling, the ontologies developed in ACCESSIBLE [16] and AEGIS ${ }^{1}$ incorporate the semantic description of solutions, applications and user interaction terms targeting users with functional limitations. Also the INREDIS Knowledge Base [11], stores all the ontologies that collect formal descriptions of the elements in the INREDIS domain (e.g. users, AS, devices, software requirements, etc.) and its instances.

Castro et al. [2] proposed the use of metadata and meta-reasoning to address the design challenges encountered when building an ontology repository for an application framework devoted to assistive technologies that can be browsed and queried in a highly heterogeneous and expressive way. They analyzed how metadata can be used in the context of open repositories of ontologies, and how it can and needs to be extended in various ways. More specifically, they studied a redesign of the Ontology Metadata Vocabulary (OMV) [5] by restructuring and enriching it with the ABC ontology [9] and domain-specific categories for assistive technologies.

The WWAAC EU project launched also an effort to use semantic web technologies for providing a generic solution for integrating the assistive technology, the web, and the signs and symbol language used traditionally by people with communication problems for text interpretation. Within the premises of the WWAAC project, the Concept Coding Framework (CCF) [10] was defined as a means to break down the isolation and barriers between different augmentative and alternative communication (AAC) symbol vocabularies by defining an open technology for connecting these vocabularies to other vocabularies and to standard lexical resources. The CCF included a plain

\footnotetext{
${ }^{1}$ http: / /www. aegis-project.eu/index.php? Itemid=65
} 
concepts list called Concept Code Definitions, the Base Reference Ontology (concepts mapped from WordNet3 to used symbols) and the Complementary Reference Ontology specifying missing concepts.

In the current work, our main purpose is to provide a high-level modeling of content-related information of ICT solutions, platforms and devices by extending and integrating previous ontological implementations. A framework that enables the semantic representation of assistive technologies is proposed, in order to encourage and enable all potential stakeholders (e.g. assistive technology owners, retailers, etc.) to use the same terms when describing the same things. Moreover, the proposed framework provides a common interface to all interested vendors, providers, etc. that intend to include their applications/solutions in the Cloud4all/GPII infrastructure $[14,15]$.

\section{The Semantic Framework for Content and Solutions (SEMA)}

The main component of the SEMA is the solutions ontology ${ }^{2}$, which aims at maintaining relevant information and metadata regarding solutions/applications, platforms, devices and their specific settings along with detailed information regarding vendors or implementers of the assistive technologies supported by the Cloud4all/GPII infrastructure. The terms inside the solutions ontology are classified according to the ISO 9999 standard [7].

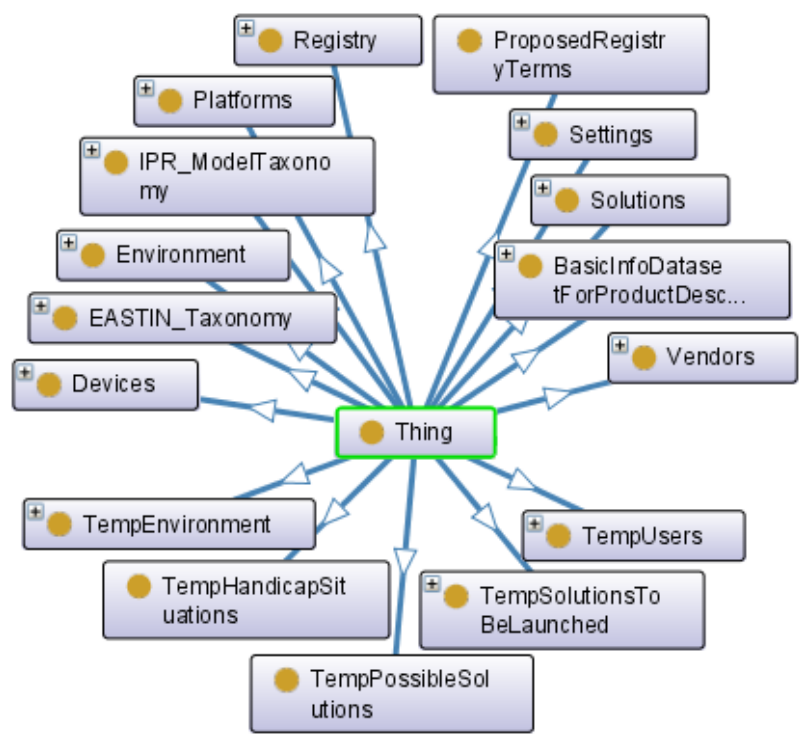

Fig. 1. Solutions ontology - Main classes

\footnotetext{
${ }^{2}$ The solutions ontology is publicly available at: http://160.40.50.183/cloud4all/
} 
Fig. 1 presents the main classes of the solutions ontology, which are the following:

- The Devices class: The Devices class and their subclasses represent in a hierarchical way the devices on which applications can run. The classification per each of the supported devices has implemented according to their domain (e.g. PC, PDA, mobile phone devices, etc.).

- The EASTIN_Taxonomy class: The EASTIN_Taxonomy class and its subclasses are used to categorize each solution according to the taxonomy proposed by the European Assistive Technology Information Network ${ }^{3}$ [1] (EASTIN), the biggest and most comprehensive information service on assistive technology serving older and disabled people, their families and careers across the globe.

- The Environment class: The Environment class and its subclasses describe the environment (ex. at home, at work) in which a solution is launched.

- The IPR_ModelTaxonomy class: The IPR_ModelTaxonomy class and its subclasses are used to describe Intellectual Property Rights (IPR), cost and trust aspects of services and applications.

- The Platforms class: The Platform class and their subclasses, represent in a hierarchical way the platforms on which solutions/applications run (e.g. Windows, Linux). A solution can be either an application or a network/cloud based service. Thus, a platform can refer either to a specific operating system on which an application runs, or to a web execution platform for web based applications and web services.

- The Registry class: The Registry class is used to store the common terms of the Registry. The Registry of common terms is a vocabulary describing common applications settings. Each instance of the class contains the definition of a Registry term.

- The ProposedRegistryTerms class: This class is used to store the common terms proposed by solution owners/vendors/retailers. The administrator of the SEMA finally decides if a suggested common term will be finally added in the Registry of common terms or it will be rejected.

- The Settings class: The term setting refers to each unique setting aspect of each solution, platform or device that can be customized according to the needs and preferences of the user. Thus, the Settings class tries to capture in a semantic manner all possible solution -, platform - and device - specific settings that could provide more added value to the personalization and adaptation process based on semantics.

- The Solutions class: The Solutions class and their descendant classes aim to classify in a semantic manner solutions/applications supported and surveyed in the Cloud4all process. The semantic classification of solutions is also based on the ISO standard 9999 "Assistive Products for Persons with Disability" 7 and includes the "subdivisions" (i.e. a refinement of the ISO 9999 standard) defined in the ETNA

\footnotetext{
${ }^{3}$ http: / /www. eastin. eu
} 
project $^{4}$. This has been selected in order to support the integration of well-known databases of ATs and accessible applications like the EASTIN.

- The BasicInfoDatasetForProductDescription class: The BasicInfoDatasetForProductDescription class is used to store some extra information such as the country of the manufacturer or the logo for each solution.

- The Vendors class: The Vendors class aims to capture and represent information about application-, platform-, and device- vendors or implementers. The Vendors class has the following subclasses: DeviceVendors, PlatformVendors and SolutionVendors.

The solutions ontology includes also a set of auxiliary classes that are essential for the proper execution of the Rule-based Matchmaker, a component of the Cloud4all/GPII infrastructure that tries to match user needs with the corresponding configurations of different assistive technologies using rules. These rules use classes and properties defined within the solutions ontology. As the rules, additionally to the solutionspecific settings, take also into account the needs and preferences of the current user as well as the current environment, the following 5 auxiliary classes had to be defined in the solutions ontology, in order to work as basic containers for storing the corresponding instances of the corresponding user and the environment.

- The TempEnvironment class: The TempEnvironment class works as a temporary container for storing the details of the user's current environment.

- The TempHandicapSituations class: The TempHandicapSituations class works as a temporary container for the description of the current problematic situations (e.g. cases where there is user preferences cannot be fully fulfilled due to the current environment/context).

- The TempPossibleSolutions class: The TempPossibleSolutions class works as a temporary container of the description of possible solutions proposed by the Rulebased Matchmaker for the settlement of the current problematic situations.

- The TempSolutionsToBeLaunched class: The TempSolutionsToBeLaunched class works as a container for storing the list of solutions that have to be launched, according to the needs/preferences of the current user and the current environment/context.

- The TempUsers class: The TempUsers class contains the corresponding temporary description of the current user

For all the 5 aforementioned auxiliary classes there are no predefined instances in the solutions ontology. Instead, temporary instances are automatically created by the Rule-based Matchmaker to enable the proper execution of the rules and they are automatically deleted after the execution of the Rule-based Matchmaker.

A variety of Datatype properties and Object properties are used to describe the instances of each class of the solution ontology in detail as well as to define relationships between different instances.

\footnotetext{
${ }^{4}$ http: / /www.etna-project.eu/
} 




Fig. 2. Alignment of application-specific settings with common terms - Example

Different applications of the same type (e.g. screen readers) usually have similar settings expressed with different names (e.g. speech-rate, voice-speed, etc.) and different value spaces (e.g. words per minute, phonemes per second, etc.). The relationship between these similar settings can be described through another component of the SEMA, the Semantic Alignment Tool ${ }^{5}$ (SAT). The SAT is a tool that acts as a middleware between the solutions ontology and the solutions/applications that are stored in the Cloud4all federated repositories and offered by different vendors. The tool's primary role is to provide a common interface to all interested vendors, providers, administrators, etc., that intend to add/modify the description of their applications/solutions as well as to align their application-specific settings with the corresponding common terms of the Registry (Fig. 2). The Registry of common terms is a vocabulary describing common applications settings (e.g., magnification factor, speech rate, font size, etc.) used to encourage and enable all stakeholders to use the same terms when describing the same things (the same concept and value range) with regard to user needs and preferences.

An application-specific setting is a setting used by a specific application while a common term of the Registry is a term that has been chosen as generic terms for a particular concept. For instance, the "magnification level" setting, which is a common setting for screen magnifiers, is defined in a different way in the Windows built-in magnifier compared with the corresponding setting of the GNOME Shell Magnifier. More specifically, the magnification level for the Windows built-in magnifier has the ID "Magnification", is an integer with value space $[100,1600]$ and its default value is 200. The magnification level for the GNOME Shell Magnifier has the ID "magfactor", is an integer with value space $[1,32]$ and its default value is 2 . Both these

\footnotetext{
${ }^{5}$ The Semantic Alignment Tool is publicly available at: http://160.40.50.183:8080/
} 
settings for magnification level can be aligned through the SAT tool to the common term of the Registry with ID "magnification", which is a float with value space $[1.0, . *$ * $]$. Fig. 3 presents a usage example of the SAT tool for aligning applicationspecific terms of different magnifiers with common terms of the Registry.



Fig. 3. Align an application-specific term to a common term of the Registry

As the manual alignment of an application-specific term with a common term of the Registry is not always a trivial task for an end user, a mechanism has been developed inside the SAT that helps user identify similar terms by performing automatic lexicographical analysis.

\begin{tabular}{l|l|}
\hline New registry term \\
Registry term name*: \\
Registry term description*: \\
Registry term value space & WordEcho each word as it is typed \\
Registry term type: & boolean \\
Registry term default value & false \\
Registry term id: & display.screenReader.-provisional-wordEcho \\
Registry term notes: & \\
Registry term alias: & Select alias \\
\hline Add & \\
\hline
\end{tabular}

Fig. 4. Proposing a new Registry term 
Moreover, through the SAT tool, a solution owner/vendor can propose new terms to be included in the Registry of common terms, as presented in Fig. 4. Then, the SEMA administrator can decide if the proposed terms should be finally included in the Registry Fig. 5.

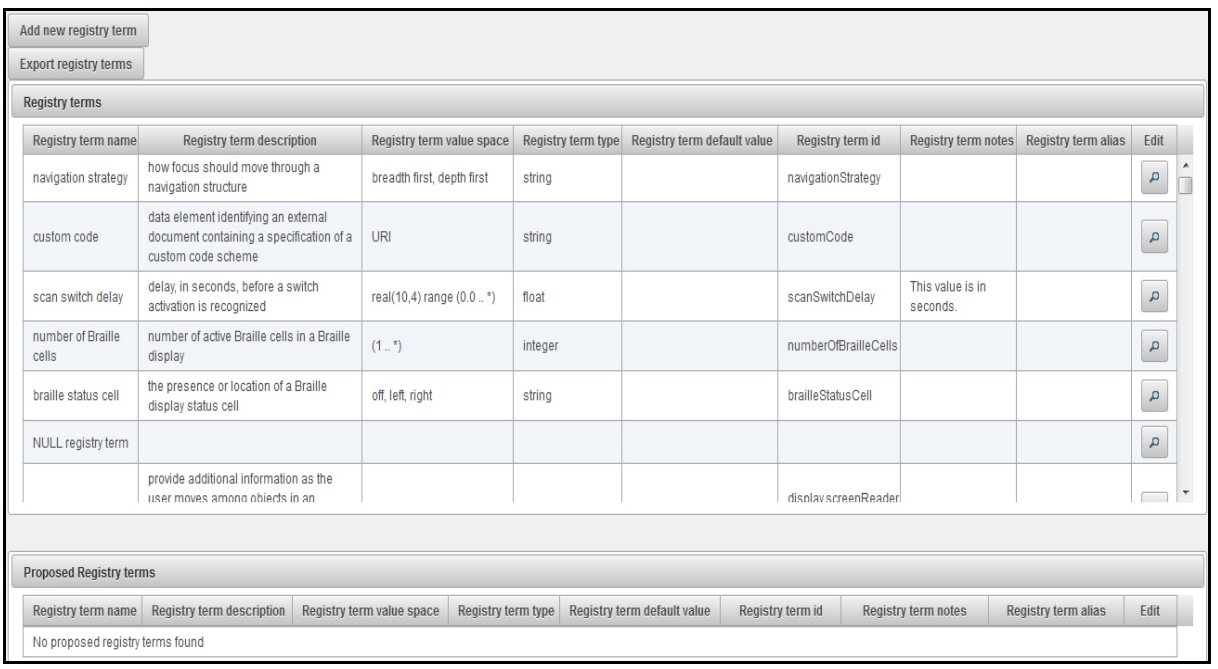

Fig. 5. Managing the Registry of common terms through the SAT tool

\section{$4 \quad$ Preliminary Results}

Currently, all the application-specific settings of the applications that were tested during the first pilot phase of the CLOUD4all project as well as the ones that will be tested during the second pilot phase have been included in the solutions ontology. Moreover, the alignment of the aforementioned settings with common terms of the Registry has been performed through the SAT tool. This knowledge was found to enhance the inference capabilities of the rule-based matchmaker and further enforce the whole process of UI adaptation for people with special needs and preferences.

\section{Conclusions}

In the present paper the Semantic Framework for Content and Solutions, a framework that enables the semantic high-level modeling of content-related information of ICT solutions, platforms and devices, was presented. The proposed framework enables the categorization of assistive technologies according to the ISO 9999 standard, the detailed description of their settings as well as the alignment between similar terms among different technologies. Moreover, it enables the integration of information coming from various external sources, such as the EASTIN databases. The SEMA provides a common interface to all interested vendors, providers, etc. that intend to 
include their applications/solutions in the Cloud4all/GPII infrastructure. Moreover, it aims at encouraging all potential stakeholders (e.g. assistive technology owners, retailers, etc.) to use the same terms when describing the same things. The Semantic Framework for Content and Solutions is a key component of the whole Cloud4all/GPII infrastructure and strengthens the UI adaptation process as it enables the detailed description of all the supported solutions, including general descriptions and metadata, and also enhances the inference capabilities of the Rule-based Matchmaker.

Acknowledgements. This work is supported by the EU co-funded project Cloud4all (FP7 - 289016).

\section{References}

1. Andrich, R.: Towards a global information network: the European Assistive Technology Information Network and the World Alliance of AT Information Providers. In: Gelderblom, G.J., Soede, M., Adriaens, L., Miesenberger, K. (eds.) Everyday Technology for Independence and Care, pp. 190-197. IOS Press, Amsterdam (2011)

2. Castro, A.G., Normann, I., Hois, J., Kutz, O.: Ontologizing Metadata for Assistive Technologies-The OASIS Repository. In: First International Workshop on Ontologies in Interactive Systems, ONTORACT 2008, pp. 57-62. IEEE (September 2008)

3. Ehrig, M.: Ontology alignment: bridging the semantic gap, vol. 4. Springer (2007)

4. Guarino, N., Masolo, C., Vetere, G.: Ontoseek: Content-based access to the web. IEEE Intelligent Systems and Their Applications 14(3), 70-80 (1999)

5. Hartmann, J., Sure, Y., Haase, P., Palma, R., Suárez-Figueroa, M.D.C.: OMV-ontology metadata vocabulary. In: ISWC 2005 Workshop on Ontology Patterns for the Semantic Web (November 2005)

6. Hildebrand, M., Van Ossenbruggen, J.: Configuring semantic web interfaces by data mapping. In: Visual Interfaces to the Social and the Semantic Web (VISSW 2009), vol. 443, p. 96 (2009)

7. ISO 9999:2011, Assistive Products for Persons with Disability - Classification and Terminology (2011)

8. Karim, S., Tjoa, A.M.: Towards the use of ontologies for improving user interaction for people with special needs. In: Miesenberger, K., Klaus, J., Zagler, W.L., Karshmer, A.I. (eds.) ICCHP 2006. LNCS, vol. 4061, pp. 77-84. Springer, Heidelberg (2006)

9. Lagoze, C., Hunter, J.: The ABC ontology and model. Journal of Digital Information 2(2) (2006)

10. Lundälv, M., Derbring, S.: AAC Vocabulary Standardisation and Harmonisation. In: Miesenberger, K., Karshmer, A., Penaz, P., Zagler, W. (eds.) ICCHP 2012, Part II. LNCS, vol. 7383, pp. 303-310. Springer, Heidelberg (2012)

11. Miñón, R., Aizpurua, A., Cearreta, I., Garay, N., Abascal, J.: Ontology-Driven Adaptive Accessible Interfaces in the INREDIS project. In: Procs. of the Int. Workshop on Architectures and Building Blocks of Web-Based User-Adaptive Systems, Haway, pp. 3739 (2010)

12. Paulheim, H., Probst, F.: Ontology-enhanced user interfaces: A survey. International Journal on Semantic Web and Information Systems (IJSWIS) 6(2), 36-59 (2010) 
13. Potter, R., Wright, H.: An ontological approach to visualization resource management. In: Doherty, G., Blandford, A. (eds.) DSVIS 2006. LNCS, vol. 4323, pp. 151-156. Springer, Heidelberg (2007)

14. Vanderheiden, G., Treviranus, J.: Creating a global public inclusive infrastructure. In: Stephanidis, C. (ed.) Universal Access in HCI, Part I, HCII 2011. LNCS, vol. 6765, pp. 517-526. Springer, Heidelberg (2011)

15. Vanderheiden, G.C., Treviranus, J., Chourasia, A.: The global public inclusive infrastructure (GPII). In: Proceedings of the 15th International ACM SIGACCESS Conference on Computers and Accessibility, p. 70. ACM (October 2013)

16. Votis, K., Lopes, R., Tzovaras, D., Carriço, L., Likothanassis, S.: A Semantic Accessibility Assessment Environment for Design and Development for the Web. In: Stephanidis, C. (ed.) UAHCI 2009, Part III. LNCS, vol. 5616, pp. 803-813. Springer, Heidelberg (2009) 